\title{
Frequency-Dependent Representation of Reinforcement- Related Information in the Human Medial and Lateral Prefrontal Cortex
}

\author{
ㅌlliot H. Smith, ${ }^{1}$ @ Garrett P. Banks, ${ }^{1}$ Charles B. Mikell, ${ }^{1}$ @Syndey S. Cash, ${ }^{2}$ Shaun R. Patel, ${ }^{3}$ Emad N. Eskandar, ${ }^{3}$ \\ and Sameer A. Sheth ${ }^{1}$ \\ ${ }^{1}$ Department of Neurological Surgery, Columbia University Medical Center, New York, New York 10032, and Departments of ${ }^{2}$ Neurology and \\ ${ }^{3}$ Neurosurgery, Massachusetts General Hospital, Boston, Massachusetts 02114
}

The feedback-related negativity (FRN) is a commonly observed potential in scalp electroencephalography (EEG) studies related to the valence of feedback about a subject's performance. This potential classically manifests as a negative deflection in medial frontocentral EEG contacts following negative feedback. Recent work has shown prominence of theta power in the spectral composition of the FRN, placing it within the larger class of "frontal midline theta" cognitive control signals. Although the dorsal anterior cingulate cortex (dACC) is thought to be the cortical generator of the FRN, conclusive data regarding its origin and propagation are lacking. Here we examine intracranial electrophysiology from the human medial and lateral prefrontal cortex (PFC) to better understand the anatomical localization and communication patterns of the FRN. We show that the FRN is evident in both low- and high-frequency local field potentials (LFPs) recorded on electrocorticography. The FRN is larger in medial compared with lateral PFC, and coupling between theta band phase and high-frequency LFP power is also greater in medial PFC. Using Granger causality and conditional mutual information analyses, we provide evidence that feedback-related information propagates from medial to lateral PFC, and that this information transfer oscillates with theta-range periodicity. These results provide evidence for the dACC as the cortical source of the FRN, provide insight into the local computation of frontal midline theta, and have implications for reinforcement learning models of cognitive control.

Key words: electrocorticography; feedback-related negativity; frontal midline theta; information representation; prefrontal cortex; reinforcement

\section{Significance Statement}

Using intracranial electrophysiology in humans, this work addresses questions about a frequently studied feedback-related electroencephalographic signal, illuminating anatomical and functional properties of the representation of feedback-related reinforcement during decision-making across the medial to lateral extent of the human prefrontal cortex.

\section{Introduction}

Modifying future behavior based on reinforcement is essential for survival in complex environments. A prominent eventrelated potential (ERP) observed on EEG is the feedback-related negativity (FRN), which manifests as the difference between ERPs evoked by positive and negative feedback (Walsh and An-

\footnotetext{
Received May 14, 2015; revised 0ct. 12, 2015; accepted 0ct. 19, 2015.

Author contributions: S.S.C., E.N.E., and S.A.S. designed research; S.S.C., S.R.P., E.N.E., and S.A.S. performed research; E.H.S., G.P.B., and C.B.M. analyzed data; E.H.S., C.B.M., and S.A.S. wrote the paper.

This work supported by NIH Grants K12 NS080223 and the Dana Foundation. We thank Jennifer Russo for help collecting data; and Lucia Melloni, Saskia Haegens, Guillermo Horga, Nima Mesgarani, and Charles Schroeder for helpful developmental advice.

The authors declare no competing financial interests.

Correspondence should be addressed to Sameer A. Sheth, 710 West $168^{\text {th }}$ Street, Room 551, New York, NY 10032. E-mail:ss4451@cumc.columbia.edu.

DOI:10.1523/JNEUROSCI.1864-15.2015

Copyright $\odot 2015$ the authors $\quad 0270-6474 / 15 / 3515827-10 \$ 15.00 / 0$
}

derson, 2012). The FRN occurs $\sim 250 \mathrm{~ms}$ after feedback, regardless of the feedback modality (Miltner et al., 1997).

The FRN is most commonly observed on frontocentral EEG contacts, where its amplitude is highest (Miltner et al., 1997; Luu et al., 2003; Silvetti et al., 2014), but has also been observed on parietal contacts (Cohen and Ranganath, 2007; Pfabigan et al., 2011). EEG and MEG source localization has commonly identified the dorsomedial prefrontal cortex (dmPFC), especially the dorsal anterior cingulate cortex (dACC), as the source of the FRN (Miltner et al., 2003; Herrmann et al., 2004; Luu et al., 2004; Roger et al., 2010; Doñamayor et al., 2011; Walsh and Anderson, 2013). fMRI studies have found areas that have increased BOLD signal for positive feedback, compared with negative feedback (Nieuwenhuis et al., 2005b). These studies have implicated a broad network in FRN generation, including cingulate cortex, dorsolateral PFC (dlPFC), the basal ganglia, and amygdalae (Hol- 
royd et al., 2004b). One recent study that combined the temporal resolution of EEG with the spatial resolution of fMRI localized the FRN to either the dACC specifically, or to a distributed frontal "salience" network, depending on the analysis technique used (Hauser et al., 2014).

An influential account of FRN function based on reinforcement learning concepts links it to the reward system (Holroyd and Coles, 2002; Holroyd et al., 2008). Mesencephalic dopamine projections to the striatum and dmPFC reinforce advantageous behaviors (Schultz, 2002). Phasic dopamine release is thought to encode reward prediction error (RPE) signals, or differences between expected and actual rewards (Schultz, 2013). Errors, resulting in negative feedback or reward omission, produce phasic decreases in dopamine release. As dopaminergic input is inhibitory to the dACC, phasic decreases disinhibit layer V dACC neurons, allowing them to depolarize and produce ERPs (Holroyd and Coles, 2002). Indeed, FRN magnitude is directly proportional to RPE magnitude and the amount of behavioral adaptation in subsequent trials (Cavanagh et al., 2010).

Recent studies have shown that the FRN, along with other frontocentral negativities, such as the error-related negativity (ERN) and N2, share common features. Spectral analyses have demonstrated increased $4-7 \mathrm{~Hz}$ power, suggesting a common mechanism of communication for these frontal midline theta

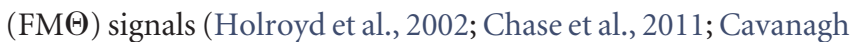
and Frank, 2014; Cavanagh and Shackman, 2015). These signals are thought to convey aspects of cognitive control and may have evolved as reactions to threatening situations, engaging cognitive resources in times of high demand (Cavanagh and Shackman, 2015).

Here we examine intracranial electrophysiology from the human dACC and dIPFC to localize and characterize the neural representation of prefrontal feedback signals. We show that the $\mathrm{dACC}$ is the source of the FRN, that the information contained in this signal propagates to dlPFC, and that this communication occurs in the theta frequency band.

\section{Materials and Methods}

Ethics statement. The Columbia University Medical Center and Massachusetts General Hospital Institutional Review Boards approved these experiments. All patients in this study provided informed consent before participating in this research.

Subjects. We examined intracranial electrocorticographic (ECoG) signals in seven patients ( 2 female) undergoing monitoring for medically refractory epilepsy. Eight-contact depth electrodes (AdTech Medical) were implanted through the mediolateral extent of prefrontal cortex (10 left hemisphere and 9 right hemisphere), thus providing simultaneous local field potential (LFP) recordings from dACC and dlPFC. Electrode placement was determined solely on clinical grounds, and participants were free to withdraw from the study without influencing their clinical care.

Depth electrode localization. Depth electrodes were localized using preoperative magnetic resonance images (MRIs) and post-implant computed tomography (CT) scans. A linear transform from CT space to preoperative MRI space was designed using FMRIB Software Library's (FSL) linear transformation algorithm (Jenkinson and Smith, 2001). Next, a transform from patient MRI space to $2 \mathrm{~mm}$ Montreal Neurological Institute standard space using both linear and nonlinear transforms was implemented (Jenkinson et al., 2005; Andersson et al., 2013). Depth electrodes' medial and lateral-most contacts were then respectively superimposed on the medial and lateral surface of the standard brain for visualization.

Behavioral task. Recordings were acquired while patients performed the Multi-Source Interference Task (MSIT; Fig. 1A; Sheth et al., 2012). The MSIT is a Stroop-like cognitive interference task requiring the sub- ject to view a stimulus consisting of three numbers (composed of integers $0-3$ ), and identify the one number ("target") different from the other two ("distracters"). The subject has to indicate his/her choice on a threebutton pad. If the target number is " 1 ," the left button is the correct choice; if " 2 ," the middle button; and if " 3 ," the right button. Importantly, the subject must choose the correct button regardless of where the target number appears in the sequence. Reaction time (RT) was defined as the time between stimulus presentation and subject response. Differences in RT among conditions was tested for significance using a oneway Kruskal-Wallis test over interference types, and a Wilcoxon signed rank test between RTs for feedback conditions.

The task contains two types of cognitive interference. Simon interference occurs because of the presence of spatial incongruence between the position of the target number in the stimulus and the position of the correct button on the button pad. For example, the correct choice for both " 200 " and " 020 " is the middle button; the first cue contains spatial interference (incongruence between the left position of the target number in the stimulus and middle position of the correct button choice), whereas the second one does not. Eriksen flanker interference occurs due to the presence of distracters that are possible button choices. For example, the correct choice for both " 133 " and " 100 " is the left button; the first cue contains distracter interference (" 3 " is a possible button choice), whereas the second one does not (" 0 " is not a possible choice).

Patients received either valenced or neutral feedback in blocks of 10 trials. During valenced feedback trials, the target number changes color to indicate whether the patient responded correctly: green for correct responses and red for incorrect responses. During neutral feedback trials, the target number changes to blue regardless of the response (Fig. 1A).

Data collection and preprocessing. Neural data from depth electrodes were sampled at $500 \mathrm{~Hz}$ on the clinical data acquisition system (Xltek, Natus Medical). First, removal of the common mode was achieved by reconstructing the data without the first principal component, based on the covariance of all examined contacts (including white matter contacts) in each patient. This method removed DC offsets, common artifacts, and line noise present in the raw data. Spectrograms were then calculated using multitapered spectral analysis with a time-bandwidth product of 5 and 9 tapers, $200 \mathrm{~ms}$ windows, $10 \mathrm{~ms}$ step sizes, and normalized relative to mean baseline spectra $(500 \mathrm{~ms}$ before the appearance of the fixation point). High gamma activity was derived from the mean values of spectrograms between 70 and $125 \mathrm{~Hz}$. These frequency limits were chosen to be above the line noise on the low end, and half of the Nyquist frequency on the upper end. To generate low-frequency ERPs, data were low-pass filtered at $40 \mathrm{~Hz}\left(150^{\text {th }}\right.$ order Fir filter $)$ and averaged over trials.

To motivate our frequency-specific hypotheses and compare this study with previous EEG studies, spectrograms and intertrial phase coherence (ITC) were calculated from Morlet wavelet decompositions on 72 scales from 1 to $125 \mathrm{~Hz}$. The group spectrogram is the average of the absolute value of each subject's spectrogram. ITC was derived from averaging the absolute value of the phase of the LFP at each frequency value in the wavelet decomposition over trials. Paired $t$ tests over trials were used to assess significant differences between feedback conditions during the outcome period for each frequency band in the time-frequency representations. These results were corrected for the number of frequency bands using the Benjamini-Hochberg false discovery rate (FDR; Hochberg and Tamhane, 1987).

FRN quantification. The FRN for both low- and high-frequency signals was defined as the difference in signal between correct and neutral feedback trials. We did not calculate the difference between correct and incorrect feedback responses because of the low error rate (see below). Previous work has shown that the FRN is dependent upon expectations established by the context, such that an FRN is observed for negative feedback when the range of outcomes varies from neutral to negative, but for neutral feedback when the range varies from positive to neutral (Holroyd et al., 2004a; Nieuwenhuis et al., 2005a; Jessup et al., 2010).

To account for variation in the size of the mean ERP between medial and lateral sites, low-frequency FRN amplitude was quantified by normalizing the maximum FRN amplitude by the ERP amplitude (i.e., the difference between the minimum and maximum) at each contact. FRN 
A

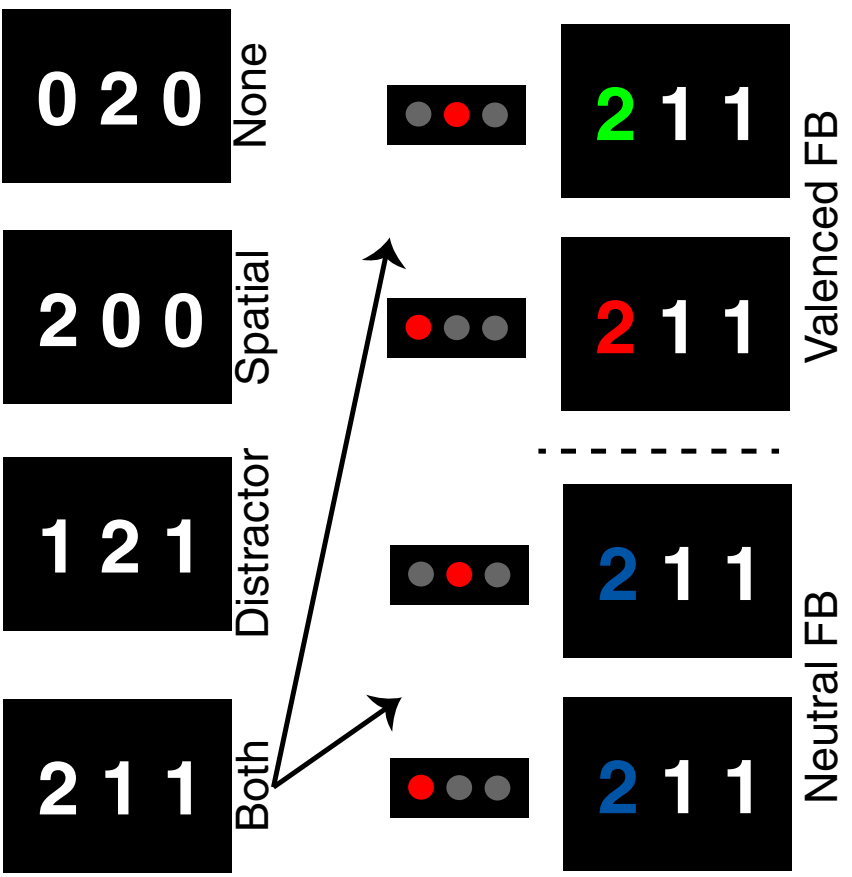

Subject

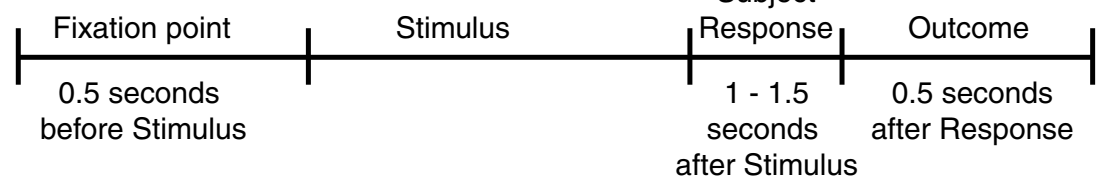

B

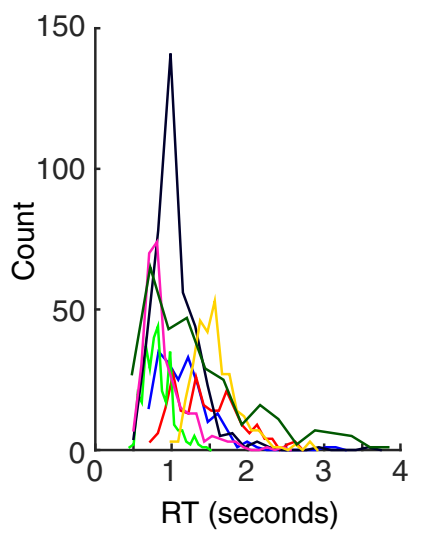

C

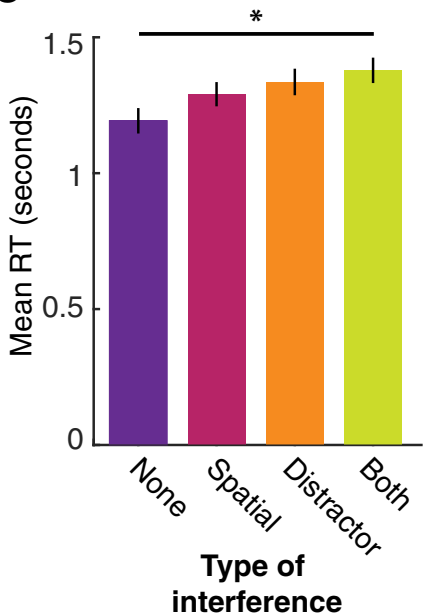

D

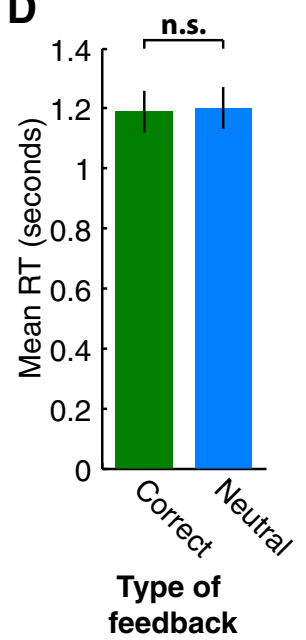

Figure 1. Task description and behavioral performance. A, MSIT task description and timeline. A fixation cross appears on the screen $500 \mathrm{~ms}$ before stimuli are shown. Four labeled examples of the different types of interference are shown above the stimulus period in the task timeline. Feedback occurs following the subject's response to the stimulus. In valenced feedback trials, the target number turns green for correct responses and red for incorrect responses. In neutral feedback trials, the target number turns blue regardless of whether the subject responded correctly. Examples of the four possible types of feedback are shown for the example stimulus that contains both types of interference. $\boldsymbol{B}$, Distributions of RTs for each subject colored by subject. $\boldsymbol{C}$, Mean RTs across subjects for trials containing the four different types of interference. The asterisk indicates statistical significance over conditions for the ANOVA. D, Mean RTs across subjects for trials following correct and neutral feedback. n.s, No significant difference.

amplitude is therefore reported relative to the size of the mean ERP at each site. For high-frequency signals, the FRN was quantified by examining differences in high gamma power during feedback presentation. Hypothesis testing for these results were performed using Mann-Whitney $U$ tests within patients and between feedback conditions for both low-frequency signals and high gamma power. To address causal influences of feedback-related representations in $\mathrm{AACC}$ and dlPFC, we calcu- lated the latency of the FRN signal as the point of separation of the averaged high gamma traces for neutral and correct feedback as long as that separation was maintained for at least $500 \mathrm{~ms}$. Latency was further quantified as the peak cross-correlation of evoked high gamma signals between medial and lateral contacts.

Phase-amplitude coupling. To determine whether there was a functional interaction between low and high-frequency LFPs we examined phase-amplitude coupling between a range of low-frequency $(2-25 \mathrm{~Hz})$ phases and highgamma power for each channel separately. The high-gamma power was determined with the Hilbert transform of the bandpass filtered signal between 70 and $125 \mathrm{~Hz}$ (4th order Butterworth). Frequencies for phase were filtered with a $3-\mathrm{Hz}$ wide bandpass filter centered on each phase frequency (4th order Butterworth). Phase values were extracted using the arctangent of the real and imaginary values of the Hilbert transforms of each filtered low-frequency band. High gamma amplitudes were then binned in 32 phase bins between $-\pi$ and $\pi$ for each low-frequency band and retained for further analysis. To determine significantly modulated phase frequencies, each phase frequency's distribution of high gamma amplitudes was tested for significant modulation with a Kolmogorov-Smirnov goodness-of-fit test against a uniform null distribution and corrected for the number of frequency bands using the FDR (Hochberg and Tamhane, 1987). The best frequency-for-phase for each electrode and patient was also determined from the Kolmogorov-Smirnov goodness-of-fit test, as the most significant frequency-for-phase band.

To quantify the amount of modulation in significant frequency-for-phase bands, modulation indices were calculated for significantly modulated frequency-for-phase bands. Modulation index was calculated in a similar manner to Canolty et al. (2006) and Miller et al. (2010, 2012). Briefly, the analytic signals returned from the Hilbert transforms of the filtered ECoG data for each trial, $n$, were separated into their amplitude and phase components $a_{n}$ and $\phi_{n}$. The modulation index used here is the magnitude of the vector defined by the complex variable as follows:

$$
z_{n}=a_{n} e^{i \phi_{n}},
$$

during the feedback period. Significance was determined by comparing distributions of these vectors projected onto the mean vector for each feedback condition using ANOVA, and the Tukey-Kramer test for pairwise comparisons.

Granger causality. Granger causality (GC) is a commonly used metric to infer directional connectivity among brain areas (Seth, 2010; Boatman-Reich et al., 2010; Barnett and Seth, 2014; Rodrigues and Andrade, 2014). GC measures the extent to which one signal can predict another by imposing time lags on one signal and examining resulting changes in the regression coefficients of an autoregressive model of both (Granger, 1969; Seth and Edelman, 2007). Here within-subject GC was determined using the Multivariate Granger Causality Toolbox for MATLAB (Barnett and Seth, 2014). We examined all possible interactions between the two medial- and lateral-most contacts among all elec- 
trodes in each patient for the feedback period. We considered interactions between combinations of pairs of electrodes in this frontal network (630 possible connections). The Lyapunov exponents of the autoregressive model based on the raw data were initially $>1$, indicating that the data did not meet the criteria of stationarity required for GC analysis. A $\mu$-law compressor, with $\mu=255$, was therefore applied to the Z-scored broadband time series to compress the dynamic range of the signals while maintaining the phase structure necessary to infer directional connectivity. The multivariate autoregressive model, $A$, henceforth had a stable spectral radius $(\rho(A)+0.96)$. Appropriate model order specification was determined using the Bayesian information criterion (Bressler and Seth, 2011). A model order of 22 was determined to be optimal for each of the seven patients and the mean \pm SD (over the 7 patients) number of lags used was $104.34 \pm 36.71$ samples.

Statistical significance was determined by testing the distribution generated by the multivariate autoregressive model against a theoretical $\chi^{2}$ distribution. The criterion for significance was set at 0.05 after correction for multiple hypotheses (Hochberg and Tamhane, 1987).

Permutation conditional mutual information. GC analysis relies on several assumptions that may not always hold in ECoG data, including stationarity, as mentioned above. Conditional mutual information (CMI) was therefore examined as an additional measure of information transfer between medial and lateral contacts. $\mathrm{CMI}$ is an information theoretic measure of information transfer between cortical areas or recording sites. CMI has been used to describe information transfer in cortical circuits without relying on Gaussian statistics or imposing time delays on neural data (Salvador et al., 2010; Quinn et al., 2011; Smith et al., 2012, 2013).

Here we computed the permutation, or symbolic, CMI (Li and Ouyang, 2010; King et al., 2013). For permutation CMI, broadband time series were discretized into groups of six symbols, three samples at a time, representing changes in the recorded voltage on each contact. Probability distributions were then generated from the pairwise frequencies of symbols derived from the two signals being compared (Keller and Lauffer, 2003; Zunino et al., 2010). CMI was then computed as follows:

$$
I(F ; X \mid Y)=\sum_{f \in F} p(f) \sum_{y \in Y} \sum_{x \in X} p(x, y \mid f) \log \frac{p(x, y \mid f)}{p(x \mid f) p(y \mid f)} .
$$

Where $F$ represents feedback, $X$ is the response in one cortical area, and $Y$ is the response in another cortical area. $p(f)$ is the probability a trial contains feedback; $p(x)$ and $p(y)$ are the probabilities of observing responses $x$ and $y$, respectively.

We calculated CMI for epochs during and just before the feedback period for medial- and lateral-most pairs of contacts on each electrode. Symbolic CMI therefore tells us how much information a signal contains about feedback valence, given that we know the response to feedback valences on the other end of the electrode. CMI regarding feedback valence was calculated for the medial electrode given that the response in the lateral electrode was known and then for the lateral electrode given that the response in the medial electrode was known. This calculation was made in $250 \mathrm{~ms}$ windows sliding $5 \mathrm{~ms}$ in time for $4 \mathrm{~s}$ surrounding feedback presentation.

We also calculated CMI for the same data with randomly shuffled samples for use in statistical comparisons. Permutation CMI is a relative measure of information transfer. Statistically significant differences from shuffled data were therefore interpreted as representing information transfer from one cortical area to the other cortical area. Statistical significance was assessed within patients using a modified $\chi^{2}$ test, comparing $\chi^{2}$ distributions generated from $2 \mathrm{Nlog}_{2}$ times the lateral-to-medial and medial-to-lateral CMI to the $\chi^{2}$ distribution from the shuffled data (Fan et al., 2000). Here $N$ is the number of trials, and the degrees of freedom for each distribution are based on the number of possible responses for each stimulus presentation and the total number of presentations (Ince et al., 2012).

To quantify the amplitude and phase of oscillations observed in the CMI signal, multitaper spectra (5 leading tapers and a time-bandwidth product of 3 ) of the CMI signals were calculated.

\section{Results}

\section{Behavioral data}

Seven subjects performed the task with similar behavior as has been previously reported, with RT increasing with amount of interference in the stimulus (Fig. 1B,C; Horga et al., 2011; Sheth et al., 2012). There was no significant difference in RT between trials with valenced versusneutral feedback (Wilcoxon signed rank, $p=0.78, N=7$; Fig. $1 D)$. Subjects had a $1.6 \pm 1.3 \%$ error rate over an average of $289 \pm 65.3$ trials per subject.

\section{Depth electrode localization}

Each depth electrode had eight contacts. In most electrodes, the two medial- and lateral-most contacts were determined to be within gray matter. The middle four contacts in each electrode were predominantly in white matter and therefore were not examined. Two medial contacts on one electrode were also determined to be in white matter. This electrode was excluded from further analysis. We therefore examined responses on 72 cortical contacts over all 7 patients. Figure 2 shows the medial and lateralmost contacts in each patient projected onto the medial and lateral surfaces of the MNI standard brain. 
A

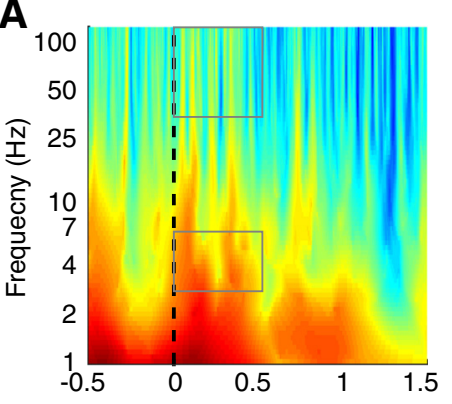

B

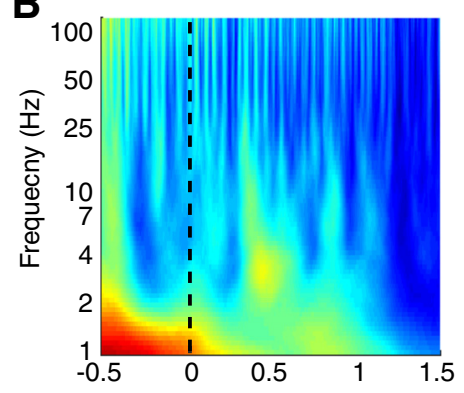

C

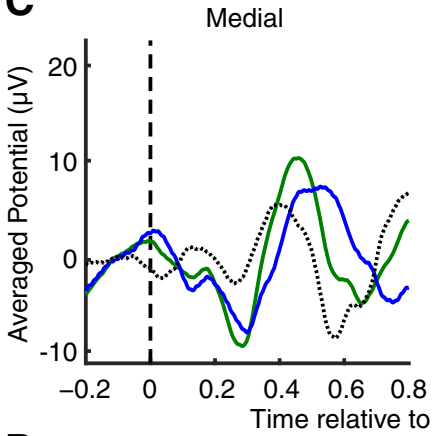

D

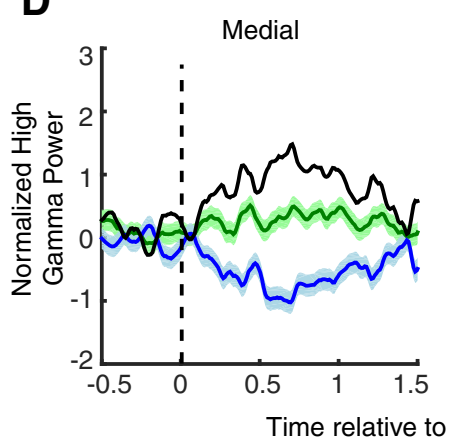

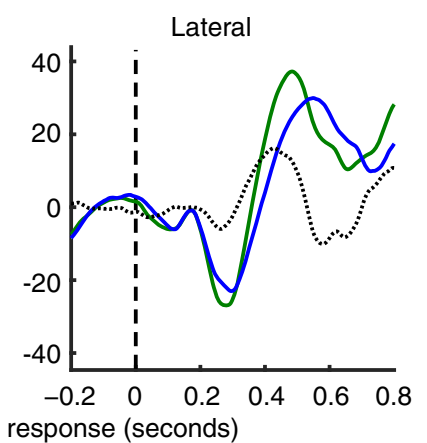

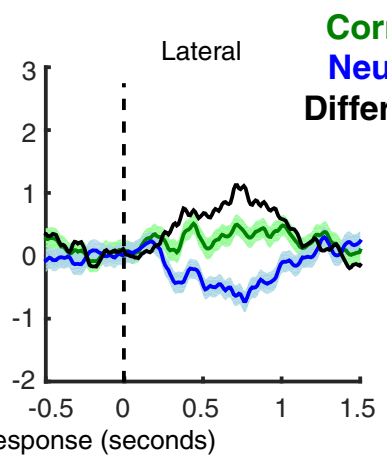

E

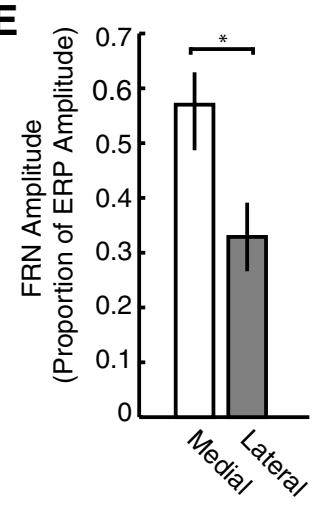

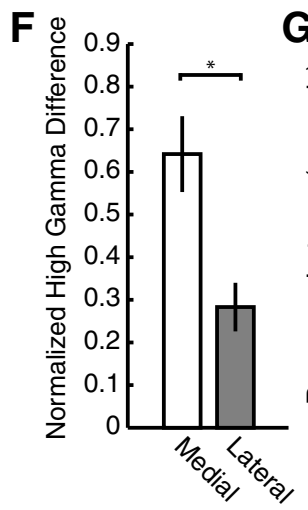

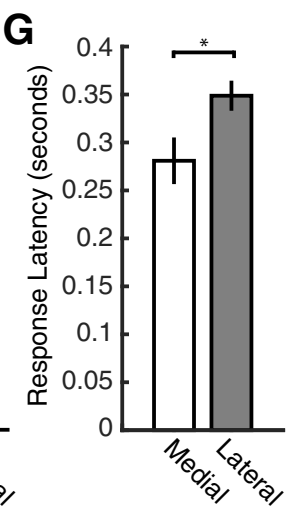

H

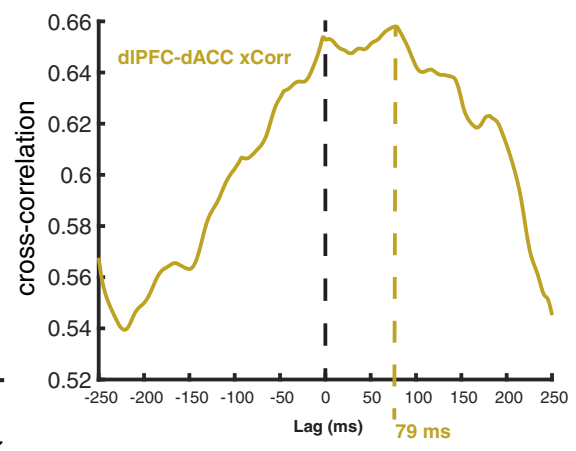

Figure 3. FRN in high- and low-frequency LFP. $A$, Response aligned spectrograms averaged over trials (mean $\pm S D=289 \pm 65.32$ ) and patients. Frequency bands during the outcome period that are significantly different between feedback conditions are outlined with gray boxes (paired $t$ tests, corrected for 72 hypotheses, all $p<0.01$ ). $\boldsymbol{B}$, Response aligned intertrial phase coherence averaged over trials and patients. $C$, Representative event related potentials from one subject for medial and lateral electrode contacts. Green and blue lines represent correct and neutral feedback conditions, respectively, and black lines represent the difference. Shading represents SE. $D$, Representative evoked high gamma response from one subject. $E$, Quantification of FRN amplitude from ERP (Mann-Whitney $U$ test, $p=0.0041, N=32$ contacts). $\boldsymbol{F}$, Quantification of high gamma power for each feedback condition (Mann-Whitney $U$ test, $p=0.00096, N=22$ contacts). $\boldsymbol{G}$, Quantification of latency differences between medial and lateral high gamma responses (two-sample $t$ test, $p=0.02, \mathrm{~N}=22$ contacts). Asterisks indicate significant differences $(p<0.05)$. $\boldsymbol{H}$, Lagged cross-correlogram between medial and lateral high gamma responses. The peak indicated that mean high gamma responses in lateral contacts lagged those in medial contacts by 79 ms.

FRN is evident in high- and low-frequency signals and is larger in dACC than dIPFC

We found evidence for differential representations of feedback valence in both low- and high-frequency components of the ECoG recordings (Fig. $3 A, B$; paired $t$ tests, all $p<0.01$ ). Figure $3 C$ depicts a representative feedback-locked ERP demonstrating the low-frequency FRN. This intracranial FRN was significant in the low-frequency averaged LFP across subjects (Kruskal-Wallis one-way ANOVA, $p \ll 0.01, \mathrm{df}=71$ contacts). There was a main effect of feedback valence in 32 of 36 medial electrodes and 32 of 36 lateral electrodes (Tukey-Kramer, all $p=0.0013, N=36$ contacts each), indicating that the FRN commonly observed on scalp EEG is evident in intracranial recordings. Low-frequency FRN was significantly greater in medial (dACC) contacts than lateral (dlPFC) contacts (Mann-Whitney $U$ test, $p=0.0041, N=$ 32 contacts; Fig. $3 E)$.
Given the relationship between high-gamma ECoG signals and local neuronal processing (Manning et al., 2009; Miller et al., 2009; Ray and Maunsell, 2011; Buzsáki et al., 2012; Einevoll et al., 2013), we also examined the high-gamma representation of the FRN in dACC and dlPFC (Fig. 3D). Feedback significantly affected high-gamma responses across subjects (Kruskal-Wallis one-way ANOVA, $p \ll 0.01, \mathrm{df}=71$ contacts). Within subjects, and across trials, there were also significant differences in evoked high-gamma power between correct and neutral feedback trials in 34 of 36 medial electrodes and 22 of 36 lateral contacts. As with low-frequency potentials, a medial-to-lateral decreasing gradient in FRN amplitude was observed in high-gamma responses (Mann-Whitney $U$ test, $p=0.00096, N=22$ contacts; Fig. $3 F$ ). Furthermore, latency of the FRN response was shorter in medial compared with lateral contacts (two-sample $t$ test, $p=0.02, N=$ 22 contacts; Fig. $3 G$ ). Cross-correlation of medial and lateral high 
gamma signals corroborated this result by estimating the mean latency as 79 ms over patients (Fig. $3 H$ ). These results indicate that the FRN is likely generated medially, in the dACC.

\section{High and low-frequency LFPs are functionally related, more so in medial PFC}

We next examined coupling between lowfrequency $(2-25 \mathrm{~Hz})$ LFP phase and highfrequency $(70-125 \mathrm{~Hz})$ LFP amplitude. We found significant phase-amplitude coupling (PAC) between theta phase and high gamma amplitude in all subjects (Kolmogorov-Smirnov goodness-offit test, all $p<0.01, N=50$ contacts each, corrected for FDR due to 23 null hypotheses; Fig. $4 A, B)$. The peak frequency-forphase was $5.1 \pm 0.2 \mathrm{~Hz}$ for correct feedback trials and $4.8 \pm 0.4 \mathrm{~Hz}$ for neutral feedback. There was no significant difference in best frequency-for-phase between medial and lateral contacts (Kruskal-Wallis one-way ANOVA, $p=$ $0.27, \mathrm{df}=71$ contacts). The magnitude of the theta-high gamma coupling, as measured by the modulation index, was significantly greater for correct feedback trials than for neutral feedback trials in medial (Tukey-Kramer, $p=0.0052, N=$ 36 contacts), but not in lateral (TukeyKramer, $p=0.0598, N=36$ contacts) contacts (Fig. 4C). In addition, the difference in theta-high gamma coupling between correct and neutral trials was greater in medial contacts than lateral contacts (Tukey-Kramer, $p=0.0098$, $N=36$ contacts; Fig. $4 E$ ). These results tie the local activity of the AACC and PFC to the theta rhythm that can be observed on scalp EEG and show that feedback information is also represented in the strength of theta-gamma coupling in dACC.

\section{Information transfer between dACC and dlPFC}

The above results provide support for the hypothesis that the FRN is generated in the dACC, and that modulation of thetagamma coupling in the dACC encodes feedback-related information. We next sought to determine the functional relationship between feedback signals across dACC and dlPFC, hypothesizing that these signals propagate from dACC to dlPFC.

To do so, we first used GC techniques to study temporal correlations between dACC and dlPFC activty. To account for the non-stationarity of ECoG data, we compressed response-aligned broadband signals on all electrodes. Both medial-to-lateral and lateral-to-medial interactions were significant $\left(\chi^{2}\right.$ test, both $p<$ $0.01, \mathrm{df}=6$, corrected for 12 hypotheses using the FDR), although GC was insignificantly greater for medial-to-lateral intercontacts).
$\mathbf{A}$

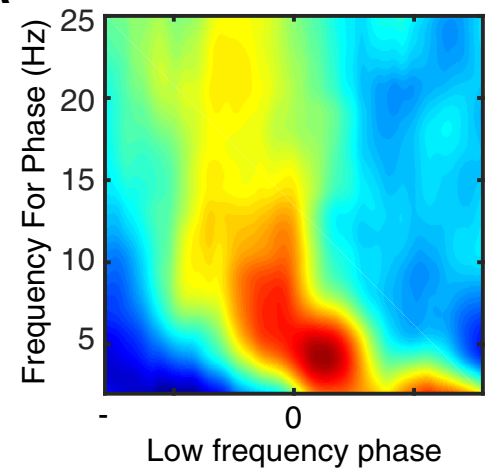

B
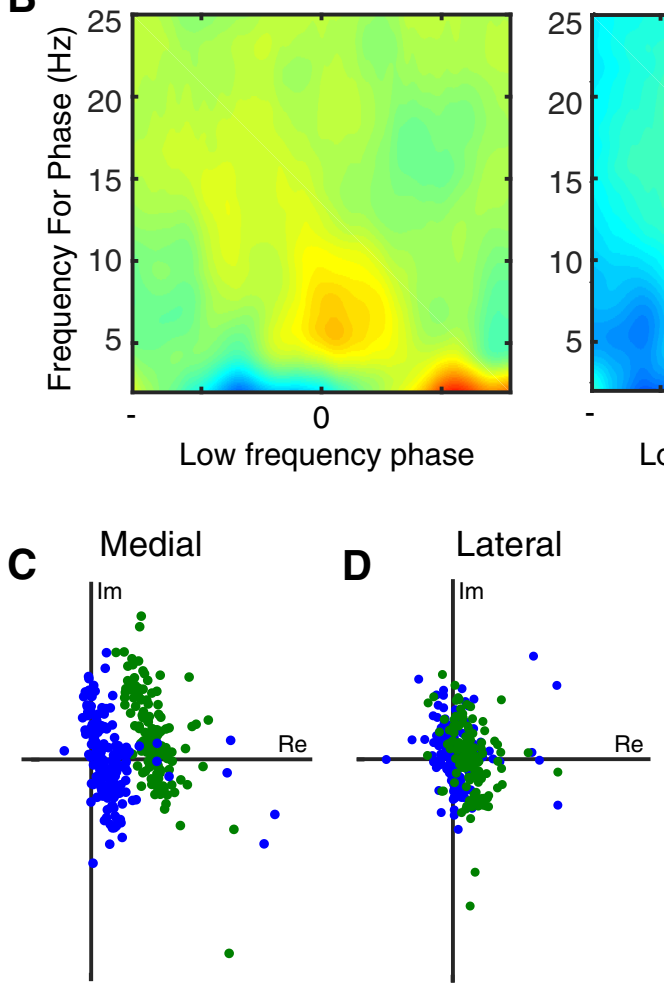

E

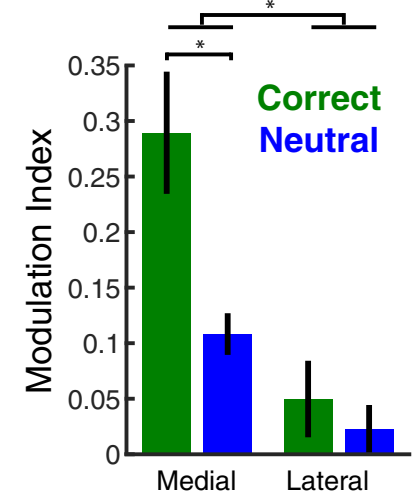

Figure 4. PAC of low- and high-frequency LFPs. $\boldsymbol{A}, \boldsymbol{B}$, Representative PAC for a medial $(\boldsymbol{A})$ and lateral $(\boldsymbol{B})$ contact in one subject for each feedback condition (correct and neutral). $\boldsymbol{C}, \boldsymbol{D}$, Plots of the real ( $x$-axis) and imaginary ( $y$-axis) components of the modulation indices over trials for a representative patient for medial $(\boldsymbol{C})$ and lateral $(\boldsymbol{D})$ contacts. Blue dots represent the modulation indices for neutral trials and green dots represent the modulation indices for correct trials. $\boldsymbol{E}$, Mean modulation indices across patients for medial and lateral contacts. Green and blue bars represent correct and neutral feedback trials, respectively. Asterisks indicate significant differences $(p<0.01)$. Modulation indices were greater for correct feedback trials than neutral feedback trials in medial (Tukey-Kramer, $p=0.0052, N=36$ contacts), but not in lateral (Tukey-Kramer, $p=0.0598, N=36$ contacts) contacts. The difference in theta-high gamma coupling between correct and neutral trials was greater in medial contacts than lateral contacts (Tukey-Kramer, $p=0.0098, N=36$

actions (Fig. 5A). Peak spectral pairwise-conditional causalities were greatest in the low theta range (mean $\pm \mathrm{SD}=4.44 \pm 0.67$ $\mathrm{Hz}$; Fig. $5 B$ ). These results suggest that there are reciprocal interactions between dACC and dlPFC.

To avoid contending with the violations of GC assumptions typical of ECoG data, we further used an analysis insensitive to these assumptions. We computed information transfer between medial and lateral PFC using a CMI approach. CMI is a singletrial measure of the reduction in uncertainty about the variable of 


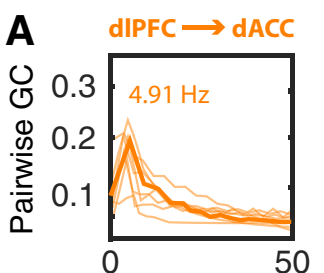

Frequency $(\mathrm{Hz})$

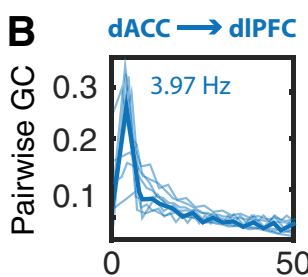

Frequency $(\mathrm{Hz})$
Figure 5. Pairwise conditional $\mathrm{GC}$ between $\mathrm{AACC}$ and $\mathrm{dIPFC}$. $A$, Pairwise $\mathrm{GC}$ over frequencies for the lateral-to-medial direction. Mean GC values across patients are shown in dark bold orange, and patient-specific data are shown in light orange. Peak frequencies averaged over patients are indicated in the colored text in the plot. $\boldsymbol{B}$, Pairwise $\mathrm{GC}$ over frequencies for the medial-to-lateral direction. Mean $\mathrm{GC}$ values across patients are shown in dark bold blue, and patient-specific data are shown in light blue. Peak frequencies averaged over patients are indicated in the colored text in the plot.

interest obtained by observing the current trial's neural data from one contact given that one knows the response on another contact. In our case, CMI is a relative measure of the dependence of feedback valence representation in one cortical area on the other cortical area. Lateral-to-medial information transfer for each subject was not significantly greater than shuffled data after feedback presentation $\left(\chi^{2}\right.$ test, all $\left.p>0.01\right)$ and did not oscillate above a constant model ( $F$ test, $F=0.288, p=0.83$; Fig. 6 ). However, CMI was significantly greater than shuffled data for medial-to-lateral information transfer for each subject $\left(\chi^{2}\right.$ test, all $p<0.01$ ), and oscillated ( $F$ test, $F=36.50, p \ll 0.01$ ) in the theta range (mean $\pm=\mathrm{SD} 5.1 \pm 2.4 \mathrm{~Hz}$; Fig. $6 A, B)$. These results support the notion of a largely unidirectional dACC-to-dlPFC transfer of feedback-related information that oscillates with theta-range periodicity.

\section{Discussion}

We examined the neural representation of prefrontal feedback signals using human ECoG with simultaneous medial and lateral PFC recordings. We report four main findings: (1) low and highfrequency ECoG signals from human PFC are sensitive to feedback valence. (2) These signals are larger and arise earlier in dACC compared with dlPFC. (3) Spectral analysis demonstrated that theta-gamma coupling modulation underlies these signals, and is also greater in dACC. (4) Information transfer analyses showed that feedback-related information is transferred from dACC to dlPFC with theta periodicity. These data thus provide direct evidence that the dACC is the cortical source of the FRN, and that theta modulation serves as a mechanism for communication of feedback-related information between dACC and lateral PFC.

\section{FRN localization: direct support for a dACC source}

Previous EEG source localization studies have posited various sites of origin for the FRN. Whereas the dACC is a common finding, others include the posterior cingulate cortex and basal ganglia (for review, see Walsh and Anderson, 2012) All these structures receive input from midbrain dopaminergic cells, making them candidate regions for producing RPE signals. Source modeling of EEG has been helpful, but the requirement of solving the inverse problem from peripherally (scalp) recorded data makes identification of a single source difficult.

Our direct human intracranial recordings demonstrated the FRN at the level of individual electrode contacts in both low- and high-frequency ranges. Although signals were seen in both dACC and lateral PFC, those from dACC were larger and had shorter
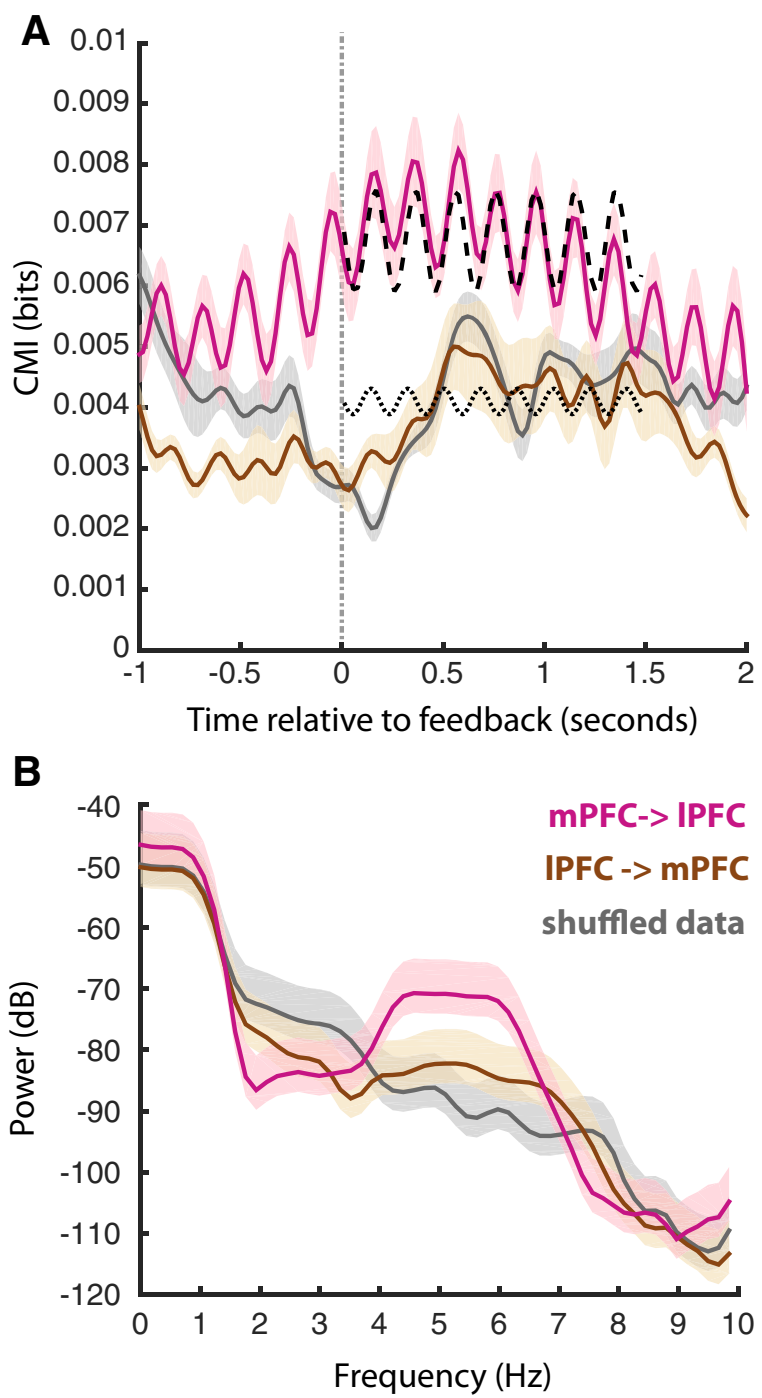

Figure 6. CMI exchange between $\mathrm{dACC}$ and $\operatorname{dIPFC}$. $\boldsymbol{A}$, Mean CMI across subjects after feedback. Pink lines represent medial-to-lateral information transfer, and brown lines represent lateral-to-medial transfer. Gray lines represent shuffled data. Dashed and dotted lines show sinusoidal fits for medial-to-lateral and lateral-to-medial CMI, respectively. Vertical line represents feedback onset. $\boldsymbol{B}$, Mean spectra of the CMI time series show increased theta power for medial-to-lateral CMI.

latencies. Human ECoG recordings have their own limitations: most importantly, that electrodes can only be placed based on clinical grounds. Because the basal ganglia are not a typical onset site for seizures, they are seldom placed there for epilepsy investigations. On the other hand, electrodes are frequently placed into the subthalamic nucleus and globus pallidus internus during deep brain stimulation procedures to treat movement disorders such as Parkinson disease and dystonia (Zavala et al., 2013,2014). Investigation of feedback responses in these nuclei in humans is therefore possible, and will be the subject of future work.

A recent combined EEG-fMRI study modeled the fMRI response informed by the EEG and also identified the dACC as the FRN source (Hauser et al., 2014). Their dynamic causal modeling analysis also showed that FRN signals were not arriving to dACC from other structures. Thus taking the current study and previous studies together, there seems little doubt that the dACC is the source of the FRN. 


\section{FRN dynamics: theta modulation}

There is increasing evidence that the FRN and similar potentials, such as the conflict-elicited N2 and the error-related negativity, share a physiological basis, namely modulation of theta power (Holroyd et al., 2004a; Cavanagh and Frank, 2014). This commonality has lead some to propose the existence of an interrelated group of cognitive control signals known as frontal midline theta $(\mathrm{FM} \Theta) . \mathrm{FM} \Theta$ is an increase in theta-range $(4-7 \mathrm{~Hz})$ spectral power observed in the event-related potentials recorded on frontocentral EEG contacts. This group of FM $\Theta$ signals is thought to use theta modulation as a means of communicating a certain class of information with other brain regions. FM $\Theta$ may be a signature of dACC activation in response to threats, either corporeal or cognitive, to signal the need for behaviorally relevant attentional control (Cavanagh and Shackman, 2015).

The current data show that the amplitude of high gamma activity, thought to be an indicator of local neuronal processing (Miller et al., 2009; Buzsáki et al., 2012), coupled to the phase of low-frequency oscillations specifically in the theta band. The modulation of this phase-amplitude coupling was sensitive to feedback valence, and was stronger in dACC than in dlPFC. Furthermore, our information transfer analyses both showed peaks in the theta range. The linear, parametric analysis, GC, indicated reciprocal interaction between $\mathrm{AACC}$ and dlPFC that was dominated by the theta range. In the context of the current study, GC has some caveats. First, that we applied a compressor to the signal to achieve stationarity and second, that the duration of the responses, especially those high-frequency responses lasting longer than $500 \mathrm{~ms}$, could bias the analyses. Conditional mutual information analysis, which is nonlinear and nonparametric, showed that feedback information arising in dACC propagated to dlPFC, and information from dlPFC did not propagate to dACC. Despite the inclusion of broadband LFP in this these analyses, the spectral composition of this information transfer showed a prominent peak centered at $5 \mathrm{~Hz}$. All of these results support the contention that the dACC uses theta range oscillations to communicate with other cognitive control centers.

Our data thus lend support to the importance of FM $\Theta$ signals in general, and to the association of the FRN with this group in particular. The conception that FRN, and FM $\Theta$, originates in the dACC, yet is represented in dIPFC is also consistent with our results. Although some EEG studies have suggested that FM $\Theta$ arises through reciprocal activation of medial and lateral prefrontal cortex (Asada et al., 1999), the intracranial electrophysiology we present indicates a largely unidirectional, medial-to-lateral transfer of feedback-related information. Another EEG study suggested that FM $\Theta$ is associated with increased theta range synchrony among increasingly lateral scalp electrodes (Cavanagh et al., 2010). However, theta power on these lateral scalp electrodes correlated with neither prediction error nor behavioral adaptation (Cavanagh et al., 2010). Our results indicating that dACC sends information to lateral prefrontal areas via a theta rhythm could explain these EEG results.

Theta oscillations have also been observed in the dACC in other species. Simultaneous single neuron and LFP recordings have enabled investigation into the mechanism and function underlying theta modulation. Previous rat (Narayanan et al., 2013) and monkey (Womelsdorf et al., 2010) studies have demonstrated that medial PFC neuronal spiking synchronizes with theta rhythms, and the magnitude of this coherence correlates with behavioral measures of cognitive control. These results argue for a mechanism by which theta-band oscillations sharpen spiking output to particular phase intervals within a theta cycle (Womelsdorf et al., 2010). This mechanism of temporal focusing may synchronize distant regions within a cognitive control network and promote more efficient spike-based communication between them.

Spike-phase coupling has been previously described as a means of integrating brain-wide networks (Fries, 2005; Lakatos et al., 2005, 2009; Womelsdorf et al., 2010). One salient example of low-frequency LFP phase coordinating neuronal population activity in distributed networks showed that prefrontal neurons are sensitive to distinct, yet diverse array of rhythms (Canolty et al., 2010). As in the current study, theta band modulation has frequently been observed in PFC (Canolty et al., 2006; Voytek et al., 2010; Miller et al., 2012; Zavala et al., 2014). Whether the dACC establishes this rhythm, or is entrained by another structure remains to be determined.

Theta rhythms are not unique to the dACC and to mechanisms of learning and cognitive control. It is known in humans and rodents that action potentials commonly couple to hippocampal theta rhythms, which are $4-8 \mathrm{~Hz}$ in the rat and $1-4 \mathrm{~Hz}$ in humans (Jacobs, 2014), and that this coupling likely supports memory processes (Buzsáki, 2005). Interestingly, action potentials couple preferentially to $4-6 \mathrm{~Hz}$ rhythms in the dACC in humans (Jacobs et al., 2007), which is the same range in which functional differences in phase amplitude coupling were observed in the current study. The medial-to-lateral CMI presented here also took on rhythmicity in precisely the 4-6 $\mathrm{Hz}$ range. These results support FM $\Theta$ arising from an information transfer architecture, as has been proposed in the rodent hippocampus (Mizuseki et al., 2009).

\section{Conclusions}

We have shown that feedback is associated with broadband ERPs and high gamma responses in the AACC and dlPFC. We have also shown that these signals are coupled to theta-band oscillations, and that information is conveyed from dACC to dlPFC entrained to a theta rhythm. Our data support a novel model for the generation of FM $\Theta$ associated with feedback in human subjects, and pave the way for future experiments to test how humans use FM $\Theta$ signals to coordinate behavioral responses to feedback or other indications for the need for cognitive control. Together, these results suggest that feedback-related information is initially processed in the AACC and quickly communicated to lateral prefrontal cortex as part of a cognitive control signal (Sheth et al., 2012; Shenhav et al., 2013). Lateral PFC presumably incorporates this information to influence ongoing behavioral modifications relevant to the immediate context and long-term goals (Miller and Cohen, 2001; Carter and van Veen, 2007).

\section{References}

Andersson JL, Jenkinson M, Smith S (2013) Non-linear registration, aka spatial normalisation. Oxford, UK: FMRIB Technical Report TR07JA2.

Asada H, Fukuda Y, Tsunoda S, Yamaguchi M, Tonoike M (1999) Frontal midline theta rhythms reflect alternative activation of prefrontal cortex and anterior cingulate cortex in humans. Neurosci Lett 274:29-32. CrossRef Medline

Barnett L, Seth AK (2014) The MVGC multivariate Granger causality toolbox: a new approach to Granger-causal inference. J Neurosci Methods 223:50-68. CrossRef Medline

Boatman-Reich D, Franaszczuk PJ, Korzeniewska A, Caffo B, Ritzl EK, Colwell S, Crone NE (2010) Quantifying auditory event-related responses in multichannel human intracranial recordings. Front Comput Neurosci 4:4. CrossRef Medline

Bressler SL, Seth AK (2011) Wiener-Granger causality: a well established methodology. Neuroimage 58:323-329.

Buzsáki G (2005) Theta rhythm of navigation: link between path integration 
and landmark navigation, episodic and semantic memory. Hippocampus 15:827-840. CrossRef Medline

Buzsáki G, Anastassiou CA, Koch C (2012) The origin of extracellular fields and currents: EEG, ECoG, LFP and spikes. Nat Rev Neurosci 13:407-420. CrossRef Medline

Canolty RT, Edwards E, Dalal SS, Soltani M, Nagarajan SS, Kirsch HE, Berger MS, Barbaro NM, Knight RT (2006) High gamma power is phaselocked to theta oscillations in human neocortex. Science 313:1626-1628. CrossRef Medline

Canolty RT, Ganguly K, Kennerley SW, Cadieu CF, Koepsell K, Wallis JD, Carmena JM (2010) Oscillatory phase coupling coordinates anatomically dispersed functional cell assemblies. Proc Natl Acad Sci U S A 107: 17356-17361. CrossRef Medline

Carter CS, van Veen V (2007) Anterior cingulate cortex and conflict detection: an update of theory and data. Cogn Affect Behav Neurosci 7: 367-379. CrossRef Medline

Cavanagh JF, Frank MJ (2014) Frontal theta as a mechanism for cognitive control. Trends Cogn Sci 18:414-421. CrossRef Medline

Cavanagh JF, Shackman AJ (2015) Frontal midline theta reflects anxiety and cognitive control: meta-analytic evidence. J Physiol Paris 109:3-15. CrossRef Medline

Cavanagh JF, Frank MJ, Klein TJ, Allen JJ (2010) Frontal theta links prediction errors to behavioral adaptation in reinforcement learning. Neuroimage 49:3198-3209. CrossRef Medline

Chase HW, Swainson R, Durham L, Benham L, Cools R (2011) Feedbackrelated negativity codes prediction error but not behavioral adjustment during probabilistic reversal learning. J Cogn Neurosci 23:936-946. CrossRef Medline

Cohen MX, Ranganath C (2007) Reinforcement learning signals predict future decisions. J Neurosci 27:371-378. CrossRef Medline

Doñamayor N, Marco-Pallarés J, Heldmann M, Schoenfeld MA, Münte TF (2011) Temporal dynamics of reward processing revealed by magnetoencephalography. Hum Brain Mapp 32:2228-2240. CrossRef Medline

Einevoll GT, Kayser C, Logothetis NK, Panzeri S (2013) Modelling and analysis of local fieldpotentials for studying the function ofcortical circuits. Nat Rev Neurosci 14:770-785. CrossRef Medline

Fan J, Hung HN, Wong WH (2000) Geometric understanding of likelihood ratio statistics. J Am Stat Assoc 95:836-841. CrossRef

Fries P (2005) A mechanism for cognitive dynamics: neuronal communication through neuronal coherence. Trends Cogn Sci 9:474-480. CrossRef Medline

Granger CWJ (1969) Investigating causal relations by econometric models and cross-spectral methods. Econometrica 37:424. CrossRef

Hauser TU, Iannaccone R, Stämpfli P, Drechsler R, Brandeis D, Walitza S, Brem S (2014) The feedback-related negativity (FRN) revisited: new insights into the localization, meaning and network organization. Neuroimage 84:159-168. CrossRef Medline

Herrmann MJ, Römmler J, Ehlis AC, Heidrich A, Fallgatter AJ (2004) Source localization (LORETA) of the error-related-negativity (ERN/Ne) and positivity (Pe). Brain Res Cogn Brain Res 20:294-299. CrossRef Medline

Hochberg Y, Tamhane AC (1987) Multiple comparison procedures. New York: Wiley.

Holroyd CB, Coles MG (2002) The neural basis of human error processing: reinforcement learning, dopamine, and the error-related negativity. Psychol Rev 109:679-709. CrossRef Medline

Holroyd CB, Coles MG, Nieuwenhuis S (2002) Medial prefrontal cortex and error potentials. Science 296:1610-1611;author reply1610-1611. CrossRef Medline

Holroyd CB, Larsen JT, Cohen JD (2004a) Context dependence of the event-related brain potential associated with reward and punishment. Psychophysiology 41:245-253. CrossRef Medline

Holroyd CB, Nieuwenhuis S, Yeung N, Nystrom L, Mars RB, Coles MG, Cohen JD (2004b) Dorsal anterior cingulate cortex shows fMRI response to internal and external error signals. Nat Neurosci 7:497-498. CrossRef Medline

Holroyd CB, Pakzad-Vaezi KL, Krigolson OE (2008) The feedback correctrelated positivity: sensitivity of the event-related brain potential to unexpected positive feedback. Psychophysiology 45:688-697. CrossRef Medline

Horga G, Maia TV, Wang P, Wang Z, Marsh R, Peterson BS (2011) Adap- tation to conflict via context-driven anticipatory signals in the dorsomedial prefrontal cortex. J Neurosci 31:16208-16216. CrossRef Medline

Ince RA, Mazzoni A, Bartels A, Logothetis NK, Panzeri S (2012) A novel test to determine the significance of neural selectivity to single and multiple potentially correlated stimulus features. J Neurosci Methods 210:49-65. CrossRef Medline

Jacobs J (2014) Hippocampal theta oscillations are slower in humans than in rodents: implications for models of spatial navigation and memory. Philos Trans R Soc Lond B Biol Sci 369:20130304. CrossRef Medline

Jacobs J, Kahana MJ, Ekstrom AD, Fried I (2007) Brain oscillations control timing of single-neuron activity in humans. J Neurosci 27:3839-3844. CrossRef Medline

Jenkinson M, Smith S (2001) A global optimisation method for robust affine registration of brain images. Med Image Anal 5:143-156. CrossRef Medline

Jenkinson M, Pechaud M, Smith S (2005) BET2: MR-based estimation of brain, skull and scalp surfaces. Paper presented at Eleventh annual meeting of the Organization for Human Brain Mapping, Toronto, Canada, June.

Jessup RK, Busemeyer JR, Brown JW (2010) Error effects in anterior cingulate cortex reverse when error likelihood is high. J Neurosci 30:3467-3472. CrossRef Medline

Keller K, Lauffer H (2003) Symbolic analysis of high-dimensional time series. Int J Bifurcation Chaos 13:2657-2668. CrossRef

King JR, Sitt JD, Faugeras F, Rohaut B, El Karoui I, Cohen L, Naccache L, Dehaene S (2013) Information sharing in the brain indexes consciousness in noncommunicative patients. Curr Biol 23:1914-1919. CrossRef Medline

Lakatos P, Shah AS, Knuth KH, Ulbert I, Karmos G, Schroeder CE (2005) An oscillatory hierarchy controlling neuronal excitability and stimulus processing in the auditory cortex. J Neurophysiol 94:1904-1911. CrossRef Medline

Lakatos P, O'Connell MN, Barczak A, Mills A, Javitt DC, Schroeder CE (2009) The leading sense: supramodal control of neurophysiological context by attention. Neuron 64:419-430. CrossRef Medline

Li X, Ouyang G (2010) Estimating coupling direction between neuronal populations with permutation conditional mutual information. Neuroimage 52:497-507. CrossRef Medline

Luu P, Tucker DM, Derryberry D, Reed M, Poulsen C (2003) Electrophysiological responses to errors and feedback in the process of action regulation. Psychol Sci 14:47-53. CrossRef Medline

Luu P, Tucker DM, Makeig S (2004) Frontal midline theta and the errorrelated negativity: neurophysiological mechanisms of action regulation. Clinical Neurophysiology 115:1821-1835. CrossRef Medline

Manning JR, Jacobs J, Fried I, Kahana MJ (2009) Broadband shifts in local field potential power spectra are correlated with single-neuron spiking in humans. J Neurosci 29:13613-13620. CrossRef Medline

Miller EK, Cohen JD (2001) An integrative theory of prefrontal cortex function. Annu Rev Neurosci 24:167-202. CrossRef Medline

Miller KJ, Sorensen LB, Ojemann JG, den Nijs M (2009) Power-law scaling in the brain surface electric potential. PLoS Comput Biol 5:e1000609. CrossRef Medline

Miller KJ, Hermes D, Honey CJ, Sharma M, Rao RP, den Nijs M, Fetz EE, Sejnowski TJ, Hebb AO, Ojemann JG, Makeig S, Leuthardt EC (2010) Dynamic modulation of local population activity by rhythm phase in human occipital cortex during a visual search task. Front Hum Neurosci 4:197. CrossRef Medline

Miller KJ, Hermes D, Honey CJ, Hebb AO, Ramsey NF, Knight RT, Ojemann JG, Fetz EE (2012) Human motor cortical activity is selectively phaseentrained on underlying rhythms. PLoS Comput Biol 8:e1002655. CrossRef Medline

Miltner WH, Lemke U, Weiss T, Holroyd C, Scheffers MK, Coles MG (2003) Implementation of error-processing in the human anterior cingulate cortex: a source analysis of the magnetic equivalent of the error-related negativity. Biol Psychol 64:157-166. CrossRef Medline

Miltner WH, Braun CH, Coles MG (1997) Event-related brain potentials following incorrect feedback in a time-estimation task: evidence for a "generic" neural system for error detection. J Cogn Neurosci 9:788-798. CrossRef Medline

Mizuseki K, Sirota A, Pastalkova E, Buzsáki G (2009) Theta oscillations provide temporal windows for local circuit computation in the entorhinalhippocampal loop. Neuron 64:267-280. CrossRef Medline 
Narayanan NS, Cavanagh JF, Frank MJ, Laubach M (2013) Common medial frontal mechanisms of adaptive control in humans and rodents. Nat Neurosci 16:1888-1895. CrossRef Medline

Nieuwenhuis S, Heslenfeld DJ, von Geusau NJ, Mars RB, Holroyd CB, Yeung N (2005a) Activity in human reward-sensitive brain areas is strongly context dependent. Neuroimage 25:1302-1309. CrossRef Medline

Nieuwenhuis S, Slagter HA, von Geusau NJ, Heslenfeld DJ, Holroyd CB (2005b) Knowing good from bad: differential activation of human cortical areas by positive and negative outcomes. Eur J Neurosci 21: 3161-3168. CrossRef Medline

Pfabigan DM, Alexopoulos J, Bauer H, Sailer U (2011) Manipulation of feedback expectancy and valence induces negative and positive reward prediction error signals manifest in event-related brain potentials. Psychophysiology 48:656-664. CrossRef Medline

Quinn CJ, Coleman TP, Kiyavash N, Hatsopoulos NG (2011) Estimating the directed information to infer causal relationships in ensemble neural spike train recordings. J Comput Neurosci 30:17-44. CrossRef Medline

Ray S, Maunsell JH (2011) Different origins of gamma rhythm and highgamma activity in macaque visual cortex. PLoS Biol 9:e1000610. CrossRef Medline

Rodrigues J, Andrade A (2014) Lag-based effective connectivity applied to fMRI: a simulation study highlighting dependence on experimental parameters and formulation. Neuroimage 89:358-377. CrossRef Medline

Roger C, Bénar CG, Vidal F, Hasbroucq T, Burle B (2010) Rostral cingulate zone and correct response monitoring: ICA and source localization evidences for the unicity of correct- and error-negativities. Neuroimage 51: 391-403. CrossRef Medline

Salvador R, Anguera M, Gomar JJ, Bullmore ET, Pomarol-Clotet E (2010) Conditional mutual information maps as descriptors of net connectivity levels in the brain. Front Neuroinform 4:115. Medline

Schultz W (2002) Getting formal with dopamine and reward. Neuron 36: 241-263. CrossRef Medline

Schultz W (2013) Updating dopamine reward signals. Curr Opin Neurobiol 23:229-238. CrossRef Medline

Seth AK (2010) A MATLAB toolbox for Granger causal connectivity analysis. J Neurosci Methods 186:262-273. CrossRef Medline

Seth AK, Edelman GM (2007) Distinguishing causal interactions in neural populations. Neural Comput 19:910-933. CrossRef Medline

Shenhav A, Botvinick MM, Cohen JD (2013) The expected value of control: an integrative theory of anterior cingulate cortex function. Neuron 79: 217-240. CrossRef Medline
Sheth SA, Mian MK, Patel SR, Asaad WF, Williams ZM, Dougherty DD, Bush G, Eskandar EN (2012) Human dorsal anterior cingulate cortex neurons mediate ongoing behavioural adaptation. Nature 488:218-221. CrossRef Medline

Silvetti M, Nuñez Castellar E, Roger C, Verguts T (2014) Reward expectation and prediction error in human medial frontal cortex: an EEG study. Neuroimage 84:376-382. CrossRef Medline

Smith EH, Kellis SS, House PA, Greger B (2012) Information transfer along the ventral auditory processing stream in the awake macaque. Conf Proc IEEE Eng Med Biol Soc 2012:5178-5181. CrossRef Medline

Smith E, Duede S, Hanrahan S, Davis T, House P, Greger B (2013) Seeing is believing: neural representations of visual stimuli in human auditory cortex correlate with illusory auditory perceptions. PLoS One 8:e73148. CrossRef Medline

Voytek B, Canolty RT, Shestyuk A, Crone NE, Parvizi J, Knight RT (2010) Shifts in gamma phase-amplitude coupling frequency from theta to alpha over posterior cortex during visual tasks. Front Hum Neurosci 4:191. CrossRef Medline

Walsh MM, Anderson JR (2012) Learning from experience: event-related potential correlates of reward processing, neural adaptation, and behavioral choice. Neurosci Biobehav Rev 36:1870-1884. CrossRef Medline

Walsh MM, Anderson JR (2013) Electrophysiological responses to feedback during the application of abstract rules. J Cogn Neurosci 25:1986-2002. CrossRef Medline

Womelsdorf T, Johnston K, Vinck M, Everling S (2010) Theta-activity in anterior cingulate cortex predicts task rules and their adjustments following errors. Proc Natl Acad Sci U S A 107:5248-5253. CrossRef Medline

Zavala B, Brittain JS, Jenkinson N, Ashkan K, Foltynie T, Limousin P, Zrinzo L, Green AL, Aziz T, Zaghloul K, Brown P (2013) Subthalamic nucleus local field potential activity during the Eriksen flanker task reveals a novel role for theta phase during conflict monitoring. J Neurosci 33: 14758-14766. CrossRef Medline

Zavala BA, Tan H, Little S, Ashkan K, Hariz M, Foltynie T, Zrinzo L, Zaghloul KA, Brown P (2014) Midline frontal cortex low-frequency activity drives subthalamic nucleus oscillations during conflict. J Neurosci 34: 7322-7333. CrossRef Medline

Zunino L, Soriano MC, Fischer I, Rosso OA, Mirasso CR (2010) Phys. Rev. E 82, 046212 (2010) Permutation-information-theory approach to unveil delay dynamics from time-series analysis. Phys Rev E Stat Nonlin Soft Matter Phys 82:046212. CrossRef Medline 